secondary care should work together towards creating clear and updated policies regarding vitamin D supplements in childhood, pregnancy and lactation, and more work on implementing these policies is needed.

\section{G295(P) PEDESTRIAN DEATHS IN IRISH CHILDREN - POTENTIAL FOR PREVENTION}

${ }^{1} \mathrm{~K}$ Hamilton, ${ }^{2} \mathrm{WL}$ Macken, ${ }^{1} \mathrm{C}$ McGarvey, ${ }^{1} \mathrm{~T}$ Matthews, ${ }^{2} \mathrm{AJ}$ Nicholson. ${ }^{1}$ National Paediatric Mortality Register, Children's University Hospital Temple Street, Dublin, Ireland; ${ }^{2}$ Department of General Paediatrics, Children's University Hospital Temple Street, Dublin, Ireland

\subsection{6/archdischild-2015-308599.272}

Aims To examine the characteristics of child pedestrian fatalities in the Republic of Ireland using the National Paediatric Mortality Register database to provide an evidence base for preventative action.

Method All child fatalities recorded on the National Paediatric Mortality Database from 1st January 2006 to 31st December 2011 were retrospectively reviewed and all road traffic related deaths were examined to identify pedestrian deaths. Passenger and cyclist deaths were excluded. Coroner's autopsy reports and death registration data were reviewed and deaths were categorised as either traffic related or non-traffic related. Deprivation scores were assigned using The Pobal Haase-Pratshke Deprivation Index.

Results There were 45 child pedestrian fatalities identified in the six year period examined. Traffic related deaths accounted for $58 \%$ vs. $42 \%$ non-traffic related. Analysis of the deaths showed there was a male preponderance. Those with a deprivation index score of "marginally below average" accounted for the majority $(53 \%)$ of deaths. There was a weekend trend and an evening and summer peak. The highest proportion of deaths occurred in the 1-4 year age group (53\%), with $28 \%$ due to low speed vehicle rollovers involving the vehicle in a reversing manoeuvre, mainly occurring in residential driveways.

Conclusion Child pedestrian fatalities are highly preventable through the modification of risk factors including behavioural, social and environmental. Most of the effort in preventing child pedestrian injuries in Ireland has focused on education of children. An area which receives little focus is non-traffic related deaths. These tragic deaths mainly involve young children/toddlers who are small in stature but independently mobile and lack the concept of personal safety. Preventative action needs to be addressed in this area.

\section{G296(P) SUPPORTING QUALITY IMPROVEMENT IN PAEDIATRICS ACROSS AN ENTIRE HEALTHCARE SYSTEM}

${ }^{1} \mathrm{~L}$ Lamb, ${ }^{1} \mathrm{G}$ Lavery, ${ }^{2} \mathrm{M}$ Smith. ${ }^{1}$ Health and Social Care Safety Forum, Public Health Agency, Lisburn, UK; ${ }^{2}$ Paediatric Department, Southern Trust, Craigavon, UK

\subsection{6/archdischild-2015-308599.273}

Aim The aim of this abstract is to describe the process of engaging and supporting those working in the paediatric service across an entire health system in a Quality Improvement (QI) programme and to describe its outcomes to date.

Methods Using the Institute for Healthcare Improvement "breakthrough collaborative" methodology, the Northern Ireland Health and Social Care Safety Forum engaged with all paediatric units to agree areas for quality improvement and to develop these into a regional driver diagram. The areas for improvement agreed upon were:

Communication - focusing on handovers, structured communications tools e.g. IPASS and SBAR, the use of safety briefings and the quality of ward rounds. The collaborative has also worked with parent representatives to develop a parent safety poster for use in all paediatric units in Northern Ireland.

Reduction in medication errors - including prescribing and administration errors

Early detection and rescue of the sick child - the collaborative have agreed a set of regional age bracketed early warning score charts and an agreed escalation protocol.

Management and Leadership - Optimising the flow of children in the acute setting

The initial three learning sessions were held over a 12 month period with action periods between. Each Trust sent a core

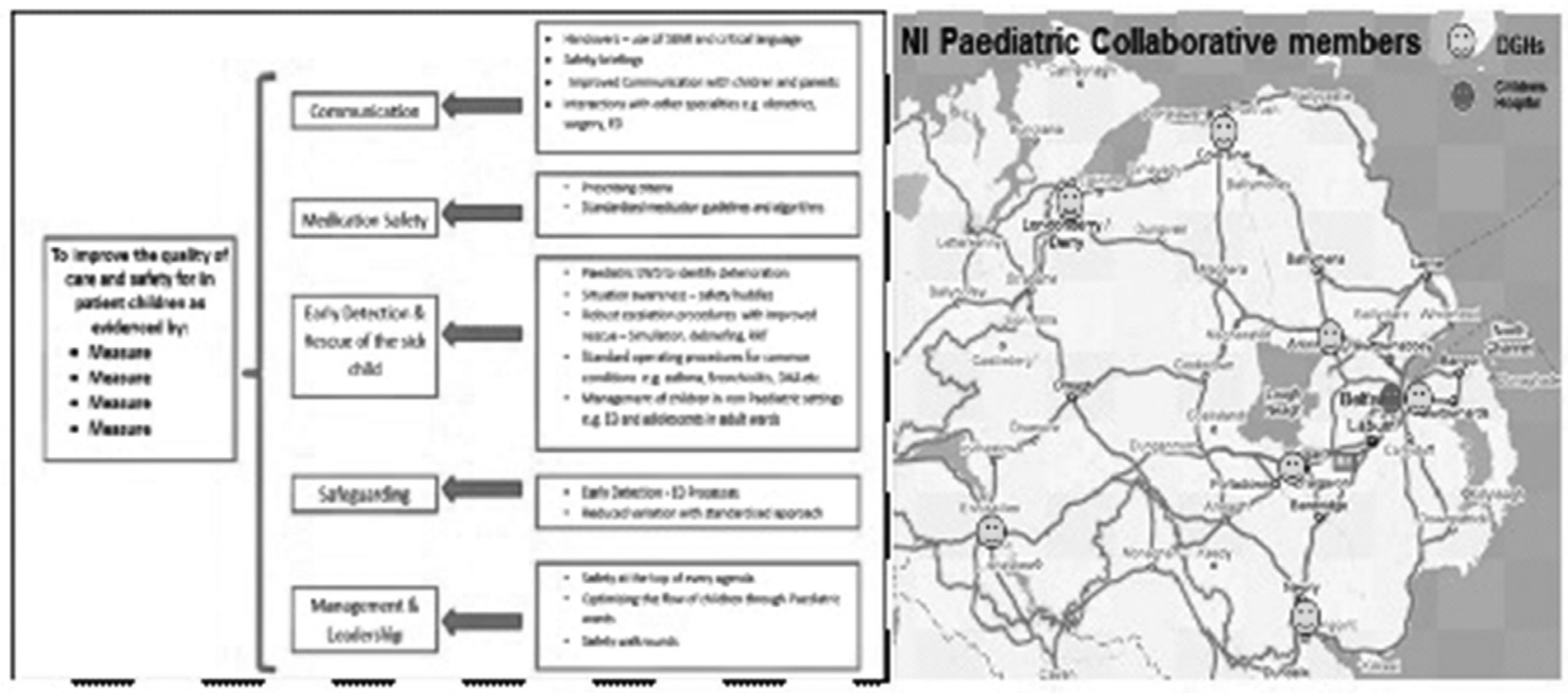

Abstract G296(P) Figure 1 Regional driver diagram and participating units 